Research report

\title{
Impact of age, sleep pressure and circadian phase on time-of-day estimates
}

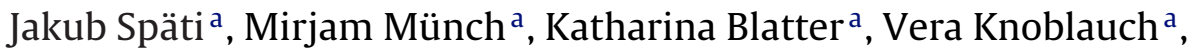 \\ Luke A. Jones ${ }^{\mathrm{b}}$, Christian Cajochen ${ }^{\mathrm{a}, *}$ \\ a Centre for Chronobiology, Psychiatric University Clinics, Basel, Switzerland \\ ${ }^{\mathrm{b}}$ Department of Psychology, University of Manchester, Manchester, UK
}

\section{A R T I C L E I N F O}

\section{Article history:}

Received 3 October 2008

Received in revised form 13 January 2009

Accepted 19 January 2009

Available online 4 February 2009

\section{Keywords:}

Self-location

Orientation

Time perception

Interval timing

Sustained wakefulness

Circadian

Core body temperature

Age

\begin{abstract}
A B S T R A C T
Orientation and self-location within the temporal fabric of the environment involves multiple organismic systems. While temporal self-location on the physiological level has been known for some time to be based on a 'biological clock' located within the hypothalamus, the mechanisms that participate in temporal position finding on the cognitive level are not yet fully understood.

In order to probe the mechanisms that underlie this faculty, verbal estimates on time-of-day were collected at 3.75-h intervals from 16 young ( 7 males, 8 females; 20 - 31 years) and 16 older ( 8 males, 8 females; 57-74 years) subjects in a balanced crossover design during 40-h epochs of prolonged wakefulness and 40-h epochs of sleep satiation spent under constant routine conditions.

An overestimation of clock time during prolonged wakefulness was found in both age-groups, with significantly larger errors for the older group (young: $0.5 \pm 0.2 \mathrm{~h}$; older: $1.5 \pm 0.2 \mathrm{~h}, p<0.05$ ). In both age-groups, estimation errors ran roughly parallel to the time course of core body temperature. However a significant interaction between time-of-day and age-group was observed (rANOVA, $p<0.05$ ): younger subjects exhibited similar estimation errors as the older subjects after $16 \mathrm{~h}$ of prior wakefulness, whereas the latter did not manifest decrements under high sleep pressure.

Data collected under conditions of sleep satiation also displayed a diurnal oscillation in estimation errors and a general overestimation (young: $0.8 \pm 0.2 \mathrm{~h}$; older: $1.3 \pm 0.3 \mathrm{~h}, p<0.05$ ). Here however, the age-groups did not differ significantly nor was there an interactive effect between time-of-day and age-group.

The effects of age, duration of wake time and circadian phase on temporal position finding are in line with predictions based on the idea that awareness about current position in time is derived from interval timing processes.
\end{abstract}

(c) 2009 Elsevier B.V. All rights reserved.

\section{Introduction}

Orientation and self-location within the temporal environment plays a critical role in any organism's adaptive behavior. A well-characterized system serving this purpose on the physiological level is the circadian master oscillator situated within the suprachiasmatic nuclei of the hypothalamus. Drawing upon transcriptional and translational feedback loops, this structure provides the organism with information about its current position within the environmental daily light-dark cycle. Sensory inputs act as 'zeitgebers', i.e., as signals controlling the synchronized run of internal and external time (circadian entrainment) [1] and, via its neural and hormonal output signals, the master oscillator allows the organism to anticipate and prepare for changes in the physical environment

\footnotetext{
* Corresponding author. Tel.: +41 61 3255318; fax: +41 613255557.

E-mail address: christian.cajochen@upkbs.ch (C. Cajochen).
}

that are associated with day and night. It thereby ensures that the organism will 'do the right thing' at the right time-of-day, and it provides internal temporal organization so internal changes take place in coordination with one another [2]. Dislocations between external time and its physiological correlate as well as alterations in the phase relationships between different physiological parameters can have detrimental health-related consequences [3]. Among the many conditions that have been linked to circadian misalignment are cardiovascular, respiratory, endocrine, rheumatological, psychiatric and neurological diseases (for references see Ref. [3]).

The mechanisms that underlie temporal self-location and orientation on the cognitive level and their potential connections to the circadian system are still poorly understood [4-6]. Whereas some species employ information about time-of-day to anticipate food availability [7] and others use time-compensated sun compass orientation to navigate long distances [8], the competence of temporal self-location on the diurnal scale has seemingly become dispensable in humans with the advent and proliferation of precise time-telling devices. Its undiminished relevance can however 
be sensed from situations in which its function is compromised. Blatant dislocations between external time and its mental representation may occur, e.g., in association with organic and functional mental disorders (such as schizophrenia or strokes) [9] and are a hallmark of dementia [10,11], but milder and transient distortions in orientation for time and temporal self-location are part of everyday life. The bewilderment we may experience upon emerging from the fictional world of an absorbing film or the fleeting moments of perplexity we may undergo upon awakening from an unscheduled nap may serve as examples. Additional relevance for the topic arises from the proliferation of working environments in which the most important of natural time cues, illumination levels, no longer ensure reliable orientation. A better understanding of the mechanisms involved in temporal self-location may also help elucidate the basis of mental time travel (imagined projection of one's temporal position to the past or the future) and, more broadly speaking, the processes that interact to produce the distinct percept of having a stable and continuous 'self' located in - and moving across - time and space.

In spite of the diversity in approaches that address the issue of cognitive temporal orientation and self-location and that range from research on time-place learning in animals [12] to anthropological psychiatry [13], models of this faculty are coarse and little is known about the mechanisms and substrates that mediate it. The establishment of quantitative theories of temporal orientation/self-location in a diurnal context (judgments about time-of-day) is complicated by the fact, that a relationship between this capacity and interval timing, i.e., the appreciation of durations in the seconds-to-hours range is often implicitly presupposed in the human literature [14]. The assumption that humans and non-human animals in some way make use of duration judgments to infer their current location in time seems plausible. However, research on time-place learning in animals $[12,15]$ and on timed awakening in humans [16] point to a critical involvement of entirely different processes in temporal self-location.

Here, we thus aimed at investigating the role of interval timing in temporal self-location. Specifically, we hypothesized that judgments about durations experienced serve a function for self-location within time which is reminiscent to the role estimates on distances travelled play for self-location within space. While navigating through space, humans and non-human animals continuously integrate cues from proprioception, inertial sensors and optical flow to infer distances covered and thus arrive at an estimate on their position relative to a starting point (spatial path integration). In moving through time, knowledge about one's current temporal location could be informed by judgments about stretches of time (i.e., durations) travelled with respect to a (temporal) starting point (temporal path integration). If this really were the case, then the inferences about temporal position drawn from duration judgments should be susceptible to factors known to modulate the latter.

Therefore, we chose to manipulate factors that are known to have a systematic impact on duration judgments and to test temporal self-location (operationalized as time-of-day judgments during temporal isolation) performed under the influence of these factors against the predictions that would ensue from the assumption of interval timing acting as sketched above (temporal path integration) in temporal position finding:

- Circadian phase: Based on the compression in subjectively perceived duration that reportedly accompanies decreased core body temperature [17], we expected subjects to underestimate the time spent in temporal isolation (and thus clock time) w.r.t. the average, during the lower part of the core body temperature cycle. Conversely, elevated core body temperature dilates a given interval's perceived duration, presumably leading to relatively positive deviations from actual clock time in time-of-day judgments.
- Duration of wake time: Increasing sleep pressure, which leads to a shortening in subjectively perceived duration [18], is expected to be reflected in a decreasing component in clock-time estimates across an epoch of extended wakefulness.

- Age: As a result of the expansion of subjectively perceived durations with age [19], we expect older subjects to display relatively more (w.r.t. the average) positive deviations in their clock-time estimates than younger subjects.

\section{Methods}

\subsection{Participants}

Sixteen healthy young ( 8 males, 8 females, mean age $25 \pm 3.5$ years, age range 20-31 years) and 16 healthy older volunteers ( 8 males, 8 females, mean age $65 \pm 5.5$ years, age range $57-74$ years) successfully completed the study. All participants were non-smokers, free from medical, psychiatric, neurologic and sleep disorders (Pittsburgh Sleep Quality Index score $\leq 5$ ) and average chronotypes (Horne-Ostberg Morningness-Eveningness score between 12 and 23) as assessed by screening questionnaires, a physical examination and a polysomnographically recorded screening night. An additional neuropsychological assessment ensured that none of the older volunteers suffered motor, attentional or memory impairments. Other exclusion criteria were: shift work within 3 months and transmeridian flights within 1 month prior to the study, excessive caffeine and alcohol consumption, drug use and excessive physical activity. Young female participants started the study on days 1-5 after menses onset in order to complete the entire study block within the follicular phase. All study participants gave signed informed consent; the local Ethics Committee approved the study protocol, screening questionnaires and consent form and all procedures conformed to the Declaration of Helsinki.

\subsection{Protocol}

The entire study consisted of two study legs of 5 days each with 2 weeks in between. During the week prior to each study leg (baseline week) participants were instructed to maintain a regular sleep-wake-cycle (bed and wake times within \pm 30 min of a self-selected target time), which was verified via wrist activity monitors (Cambridge Neurotechnologies, UK) and sleep logs. The two study legs comprised two conditions: high sleep pressure (SD protocol) and low sleep pressure (NAP protocol), which were conducted in a balanced crossover design. Each study leg consisted of an adaptation night and a baseline night, followed by $40 \mathrm{~h}$ of either sleep deprivation or sleep satiation, and a recovery night. The sleep-wake schedules were calculated by centering the $8 \mathrm{~h}$ sleep episodes on the midpoint of each individual's habitual sleep time as assessed by actigraphy and sleep logs during the baseline week. Low sleep pressure was attained using 10 alternating cycles of 75 min of scheduled sleep (naps) and 150 min of scheduled wakefulness. In both the SD and the NAP protocol, wake episodes were spent under constant routine conditions (constant dim light levels, $<8 \mathrm{~lx}$, semi-recumbent posture in bed, food and liquid intake at regular intervals, no cues on time-of-day. During scheduled sleep episodes, a minor shift to supine posture was allowed and lights were turned off (0 lx, for more information see Ref. [20].

\subsection{Measures}

- Temporal orientation: Cognitive temporal orientation was assessed across the $40 \mathrm{~h}$ constant routine episode by prompting a verbal estimate about time-of-day every $3.75 \mathrm{~h}$ (centered within the phases of wakefulness during NAP and at equivalent positions during SD). A lab technician prompted the estimate via an interphone by asking the subject the standard question: "What time is it now according to your opinion?" Subjects were instructed to respond spontaneously and to avoid calculations or comparable cognitive strategies to infer actual clock time. We used a set interval as opposed to randomized intervals to sample time-of-day judgments in order to keep possible masking effects constant.

- Core body temperature (CBT): CBT was recorded at 20-s intervals using an indwelling rectal probe (Interstar, Cham, Switzerland; Therm, type 5500-3, Ahlborn, Holzkirchen, Gemany). The temperature was displayed on a personal computer screen and continuously monitored by a lab technician. After the study, the recordings were visually inspected and artifacts resulting from removal or malfunction of the probe were excluded from further analysis.

\subsection{Statistical analyses}

Mean temporal orientation errors were calculated based on individual differences between clock-time estimate and actual clock time; one participant from the younger group (male, 28 years) had to be excluded from further analyses because he did not comply with the test procedure. Statistical analyses of the time course in temporal orientation errors and core body temperature respectively, were carried out using ANOVA for repeated measures (rANOVA) with Huynh-Feldt's statistics and Curran Everett's alpha-corrected $t$-test for post hoc tests. Correlations between 

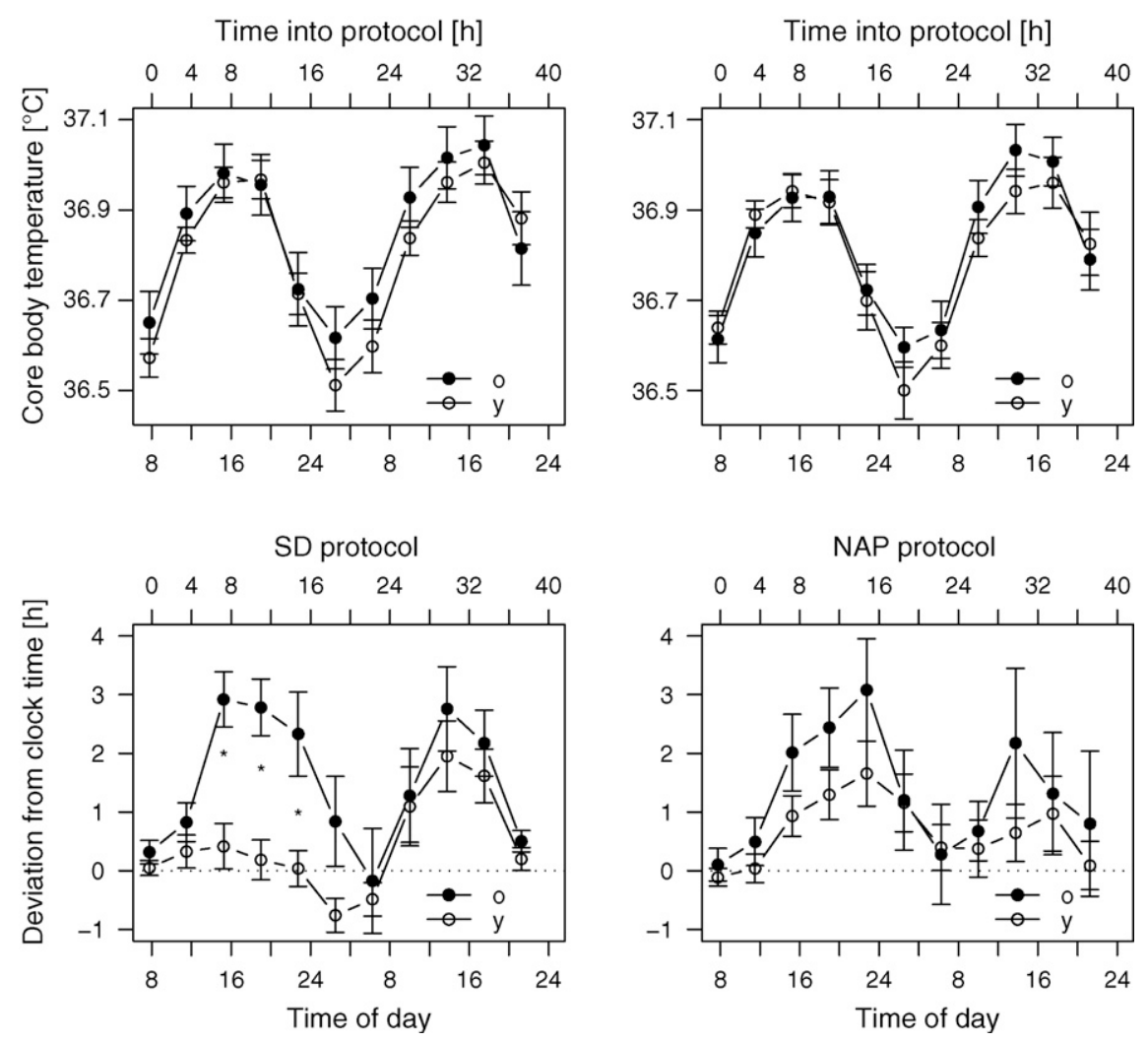

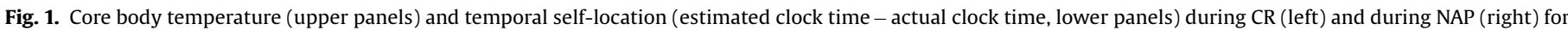
older (filled circles) and younger (open circles) subjects. Data were binned in 3.75-h intervals, mean \pm S.E.M., $n=15$ for young and $n=16$ for older subjects.

temporal orientation errors and core body temperature were calculated on an individual basis and separately for the CR and the NAP protocols.

The alpha-criterion was set at $p=0.05$. The statistical packages $\mathrm{R}$ (The R Foundation for Statistical Computing, Vienna, Austria; Version 2.7.0), SAS (SAS Institute, Inc., Cary, NC; Version 6.12), and STATISTICA (StatSoft Inc., Tulsa, OK; Version 6.1) were used.

\section{Results}

\subsection{Core body temperature}

A three-way rANOVA on the CBT data using the factors "sleep pressure", "age-group" and "time-of-day" yielded significance for the factor "time-of-day" $(F(10,290)=57.73, p<0.001)$, but no further significant effects or interactions.

\subsection{Temporal orientation}

A five-way rANOVA using the factors "age-group", "gender", "order of protocols" and the repeated factors "time-of-day" and "sleep pressure" (SD/NAP) on mean estimation errors revealed significant effects for the factors "time-of-day" $(F(10,230)=3.5$, $p<0.001)$ and "age-group" $(F(1,23)=4.5, p<0.05)$ and a significant interaction of the factors "time-of-day" and "sleep pressure" $(F(10,230)=5.0, p<0.001)$. Other factors, including the effect of protocol order and gender did not yield significance. Separate fourway rANOVAs for the young and the older age-group, respectively, yielded no significant effect for the factors "gender", "sleep pressure" and "order of protocols" on mean estimation errors:

- Sleep satiation (NAP): Under conditions of sleep satiation (NAP), young and older subjects generally overestimated time-of-day (young: $0.8 \pm 0.2 \mathrm{~h}$; older: $1.3 \pm 0.3 \mathrm{~h}$ ). This overestimation of clock time did not differ significantly between age-groups, but indicated a strong temporal correlation with the time course of CBT for both age-groups (Fig. 1, bottom right panel, factor "timeof-day": $F(10,290)=3.5, p<0.000)$ with low (close to zero) values during the CBT minimum and high values (up to $3 \mathrm{~h}$ ) during the plateau of the CBT maximum.

- Prolonged wakefulness (SD): During SD we found an average overestimation of clock time in both age-groups, with significantly higher values for older subjects (Fig. 1, bottom left panel, young: $0.5 \pm 0.2 \mathrm{~h}$; older: $1.5 \pm 0.2 \mathrm{~h}$, factor "age-group": $F(1,29)=5.6$, $p=0.025)$. Estimation errors varied in a diurnal fashion in both age-groups $(F(10,290)=8.3, p<0.001)$, the oscillation running roughly parallel to the time course of CBT (Fig. 1, top left panel). Furthermore, a significant interaction of the factors "time-ofday" and "age-group" was found $(F(10,290)=2.7, p=0.04)$. Post hoc analysis on the SD data revealed significantly larger estimation errors for older subjects after $8.6 \mathrm{~h}$ of prior wakefulness (corresponding time-of-day $16.6 \mathrm{~h}$ ), after $12.4 \mathrm{~h}$ (corresponding time-of-day $20.4 \mathrm{~h}$ ) and after $16.1 \mathrm{~h}$ (corresponding time-of-day $24.1 \mathrm{~h}$ ). Beyond $16.1 \mathrm{~h}$ of elapsed time into protocol, the mean estimation error found in the younger group increased up to the level present in the older group.

Analysis of the correlation between temporal orientation errors and core body temperature on average yielded a higher correlation for the CR condition (mean: 0.60 ; range: -0.39 to 0.81 ) than for the NAP condition (mean: 0.22; range: -0.67 to 0.09 ).

\section{Discussion}

During $40 \mathrm{~h}$ of constant posture, ambient light and temperature conditions and without information about time-of-day, awareness 
about temporal position is clearly affected by circadian phase and duration of wake time (i.e., sleep pressure) and it is generally impaired in older participants. These findings militate in favour of the idea of interval timing processes participating prominently in temporal self-location, because critical features of the data are in line with predictions that ensue from this hypothesis:

- Circadian phase: The diurnal modulation in estimation errors and its striking synchronicity with the daily core body temperature cycle (particularly under CR conditions) provide strong support for an interval timing basis of self-location. A given interval is known to subjectively appear compressed or dilated as a function of lowered or elevated core body temperature, respectively $[21,22,17]$, and the intrinsic diurnal oscillation in core body temperature - which is ultimately driven by the circadian master clock [23]- thus entails a synchronous oscillation in the appreciation of temporal intervals $[24,25]$. We argue that this diurnal oscillation in duration judgments in turn plays a pivotal role in the observed under- and overestimations of actual clock time, as it may cause a distortion in perceived 'temporal distance' travelled from the last 'temporal landmark', i.e., the last indexed (i.e., cognitively appreciated) point in time.

- Duration of wake time: The wake-time dependency in estimation errors observed in younger subjects are more difficult to conciliate with this idea. Previous studies on the effect of sleep deprivation on interval timing yielded inconclusive results, but they suggest a compression of perceived durations with prolonged wakefulness [18], which would entail increasingly negative (w.r.t. to the baseline) errors in temporal self-location across the SD protocol, rather than the increase observed in our data. Why this effect is seen in the younger subjects solely is a complex question. Interestingly, psychomotor vigilance task (PVT) data collected within the same study, displayed a number of similar features for reaction times assessed under low and high sleep pressure conditions [26]. Older subjects displayed slower reaction times during the biological day in the NAP protocol, and during the first $16 \mathrm{~h}$ in the SD protocol. An observed PVT performance decline after SD 'was significantly less pronounced in the older than in the young subjects, so that both age-groups exhibited similar performance decrements after $16 \mathrm{~h}$ into the SD protocol' [26], a feature that is in support of previous findings on the differential impact of sleep deprivation on reaction time measures in different age-groups [27-29]. The significance for temporal orientation of this strikingly similar response of PVT measures in the face of sleep deprivation remains to be elucidated but could indicate an overlap in functional components of the mechanisms subserving these outputs and could relate to the reduced effect of sleep loss in the elderly due to a loss in profound build-up of homeostatic sleep pressure as indexed by a reduced relative increase of frontal EEG delta activity in the elderly during recovery sleep' $[26,30]$. Another point worth considering is the possibility that the differences found between SD and NAP are due to an interfering effect of the interspersed sleep episodes in NAP vs. the lack thereof in SD per se, rather than the resulting difference in sleep pressure. It is conceivable, that the regular spacing of sleep episodes during NAP provided time cues absent in SD or that a state of sleep inertia impacted duration judgments made after naps. We tried to avoid the latter problem by centering the sampling points within the wake phases during NAP and, while a possible 'pacemaker effect' of the naps during NAP cannot be excluded, a timing signal was in principle given equally for both NAP and SD conditions by the prompts on time-of-day judgments themselves.

- Age: The age-related differences in average estimation errors present in our data again back the notion of temporal path integration. Several studies have reported an expansion of subjec- tively perceived durations in older subjects, which is consistent with the larger overestimation of clock time found in this agegroup.

The circadian and age-related effects observed in our data are similar to those described in a study by Campbell et al.[14]. In this study, 69 subjects living in temporal isolation for $72 \mathrm{~h}$ with freely chosen sleep episodes gave estimates on time at irregular intervals (mean interval $=3.1 \mathrm{~h}$; S.D. $=0.85 \mathrm{~h}$ ), from which the authors derived the measure of a mean subjective hour within a given interval. Although the overall average subjective hour for the entire group in this study lasted for $67.8 \mathrm{~min}$ (i.e., for each elapsed hour, subjects experienced the passage of only $53.1 \mathrm{~min}$ ) which - in contrast with our findings - reflects an overall underestimation of clock time, the data revealed a significant effect of age with shorter subjective hours for older subjects and a diurnal modulation of the subjective hour with a relatively longer duration within the lower portion of the temperature cycle (which translates into the perception that physical time is passing at a relatively faster rate and shorter estimates) and and a relative compression of the subjective hour (which translates into the perception that physical time is passing at a relatively slower rate and longer estimates) at the upper part of the temperature cycle, two features that are consistent with our data. On the other hand, Aschoff [24] found a close temporal relationship between the estimation of time intervals in the seconds range with the circadian time course of core body temperature but no effect of the duration of wake time (but see Ref. [18]; paragraph about duration of wake-time effects above). In contrast, perception of intervals in the hours range correlated positively with the duration of wake time but not with body temperature in the findings reported by Aschoff. These discrepancies are most likely due to the fact that in Aschoff's study, subjects were asked to produce time intervals whereas in Campbell's and our's studies interval timing measures were derived from time-ofday judgments which may fall into a completely different class of phenomena. The overall underestimation of clock time described by Campbell has been reported in number of other studies on temporal orientation and is in contrast with what we found for our sample; we believe however, that the general overestimation present in our data can be explained in terms of the experimental setting. It is a well-known feature of duration judgments that a duration appears to be dilated in retrospect, if the interval to be judged is filled with a rapid succession of novel events. In our study, and contrasting with earlier work on temporal orientation, subjects experienced frequent contextual change due to the fastpaced sequence of events (snacks, saliva samples, neurocognitive testing, etc.) imposed by the protocol which, via exaggerated retrospective duration judgments, could have lead to the observed overestimations.

In spite of the aforementioned caveats, we are thus confident that our hypothesis can be fruitfully applied to the study of temporal orientation and that alternative explanations of temporal self-location under constant routine conditions (i.e., inferences from beard growth, information on the state of the circadian clock pushing into cognition) either yield predictions inconsistent with the empirical data or would require a number of additional assumptions in order to achieve the same predictive power as the concept of temporal path integration. As we followed a purely correlative approach here to back our hypothesis, we suggest further investigation of this topic using experimental interventions, e.g., active manipulation of core body temperature and even more careful control for possibly confounding variables (spacing of prompts, sleep episodes, etc). Experiments of this kind could eventually provide us with a much clearer view on cognitive temporal orientation than is currently available. 


\section{Acknowledgements}

We thank Claudia Renz, Marie-France Dattler and Giovanni Balestrieri for their help in data acquisition, Drs. Carmen Schröder and Corina Schnitzler for the medical screening and coverage, Sylvia Frey and Dr. Peter Steck for their helpful comments and the subjects for participating. This research was supported by Swiss National Foundation Grants START \#3130-054991.98 and \#3100-055385.98 to CC and the Velux Foundation (Switzerland).

\section{References}

[1] Moore MD, Robert Y. Circadian rhythms: basic neurobiology and clinical applications. Annu Rev Med 1997;48(1):253-66.

[2] Vitaterna MH, Takahashi JS, Turek FW. Overview of circadian rhythms. Alcohol Res Health 2001;25:85-93.

[3] Rajaratnam SM, Arendt J. Health in a 24-h society. Lancet 2001;358(9286): 999-1005.

[4] Macleod RB, Roff MF. An experiment in temporal disorientation. Acta Psychol 1936; $1: 381-423$

[5] Thor DH, Crawford MLJ. Time perception during a two-week confinement: influence of age, sex, IQ, and time of day. Acta Psychol 1964;22:78-84.

[6] Vernon JA, McGill TE. Time estimations during sensory deprivation. J Gen Psychol 1963;69:11-8.

[7] Pizzo MJ, Crystal JD. Representation of time in time-place learning. Anim Learn Behav 2002;30:387-93.

[8] Reppert SM. The ancestral circadian clock of monarch butterflies: role in timecompensated sun compass orientation. Cold Spring Harb Symp Quant Biol 2007;72:113-8.

[9] Joslyn D, Hutzell RR. Temporal disorientation in schizophrenic and braindamaged patients. Am J Psychiatry 1979;136:1220-2.

[10] Berrios GE. Orientation failures in medicine and psychiatry: discussion paper. J Roy Soc Med 1983;76:379-85.

[11] Giannakopoulos P, Gold G, Duc M, Michel JP, Hof PR, Bouras C. Neural substrates of spatial and temporal disorientation in Alzheimer's disease. Acta Neuropathol 2000;100:189-95.

[12] Carr JAR, Wilkie DM. Ordinal, phase, and interval timing. In: Bradshaw CM, Szabadi E, editors. Time and behaviour psychological and neurobehavioural analyses, vol. 120. North-Holland; 1997. p. 265-327 [Chapter 7].

[13] Lehmann HE. Time and psychopathology. Ann NY Acad Sci 1967;138:798-821 [Interdisciplinary perspectives of time].
[14] Campbell SS, Murphy PJ, Boothroyd CE. Long-term time estimation is influenced by circadian phase. Physiol Behav 2001;72(4):589-93.

[15] Van der Zee EA, Havekes R, Barf RP, Hut RA, Nijholt IM, Jacobs EH, et al. Circadian time-place learning in mice depends on cry genes. Curr Biol 2008; 18 : 844-8.

[16] Aritake S, Uchiyama M, Tagaya H, Suzuki H, Kuriyama K, Ozaki A, Tan X, Shibui $\mathrm{K}$, Kamei Y, Okubo Y, Takahashi K. Time estimation during nocturnal sleep in human subjects. Neurosci Res 2004;49(4):387-93.

[17] Wearden JH, Penton-Voak IS. Feeling the heat: body temperature and the rate of subjective time, revisited. Quart J Exp Psychol B 1995;48: 129-41.

[18] Miro E, Cano MC, Espinosa-Fernandez L, Buela-Casal G. Time estimation during prolonged sleep deprivation and its relation to activation measures. Hum Factors 2003;45:148-59.

[19] Block RA, Zakay D, Hancock PA. Human aging and duration judgments: a metaanalytic review. Psychol Aging 1998;13:584-96.

[20] Cajochen C, Knoblauch V, Kräuchi K, Renz C, Wirz-Justice A. Dynamics of fronta EEG activity, sleepiness and body temperature under high and low sleep pressure. Neuroreport 2001;12:2277-81.

[21] Hoagland $H$. The physiologic control of judgments of duration: evidence for a chemical clock. J Gen Psychol 1933;9:267-87.

[22] Bell CR. Effects of lowered temperature on time estimation. Quart J Exp Psychol 1975;27:531-8.

[23] Kräuchi K. How is the circadian rhythm of core body temperature regulated? Clin Auton Res 2002;12:147-9.

[24] Aschoff J. Human perception of short and long time intervals: its correlation with body temperature and the duration of wake time. J Biol Rhythms 1998; 13:437-42.

[25] Pöppel E, Giedke H. Diunal variation of time perception. Psychol Forsch 1970;34:182-98

[26] Blatter K, Graw P, Münch M, Knoblauch V, Wirz-Justice A, Cajochen C. Gender and age differences in psychomotor vigilance performance under differential sleep pressure conditions. Behav Brain Res 2006;168(2):312-7.

[27] Bonnet MH. The effect of sleep fragmentation on sleep and performance in younger and older subjects. Neurobiol Aging 1989;10(1):21-5.

[28] Philip P, Taillard J, Quera-Salva MA, Bioulac B, Akerstedt T. Simple reaction time, duration of driving and sleep deprivation in young versus old automobile drivers. J Sleep Res 1999;8:9-14.

[29] Philip P, Taillard J, Sagaspe P, Valtat C, Sanchez-Ortuno M, Moore N, et al. Age performance and sleep deprivation. J Sleep Res 2004;13:105-10.

[30] Münch M, Knoblauch V, Blatter K, Schröder C, Schnitzler C, Kräuchi K, et al. The frontal predominance in human EEG delta activity after sleep loss decreases with age. Eur J Neurosci 2004;20:1402-10. 